Draft version April 30, 2018

Preprint typeset using $\mathrm{LAT}_{\mathrm{E}} \mathrm{X}$ style emulateapj v. 12/16/11

\title{
SPECKLE INTERFEROMETRY AT SOAR IN 2016 AND 2017
}

\author{
ANDREI TOKOVININ \\ Cerro Tololo Inter-American Observatory, Casilla 603, La Serena, Chile
}

Brian D. Mason \& William I. Hartkopf

U.S. Naval Observatory, 3450 Massachusetts Ave., Washington, DC, USA

Rene A. Mendez

Universidad de Chile, Casilla 36-D, Santiago, Chile

\author{
Elliott P. HORCH* \\ Department of Physics, Southern Connecticut State University, 501 Crescent Street, New Haven, CT 06515, USA \\ Draft version April 30, 2018
}

\begin{abstract}
The results of speckle interferometric observations at the $4.1 \mathrm{~m}$ SOAR telescope in 2016 and 2017 are given, totaling 2483 measurements of 1570 resolved pairs and 609 non-resolutions. We describe briefly recent changes in the instrument and observing method and quantify the accuracy of the pixel scale and position angle calibration. Comments are given on 44 pairs resolved here for the first time. Orbital motion of the newly resolved subsystem BU $83 \mathrm{Aa}, \mathrm{Ab}$ roughly agrees with its 36 year astrometric orbit proposed by J. Dommanget. Most Tycho binaries examined here turned out to be spurious.
\end{abstract}

Keywords: stars: binaries

\section{INTRODUCTION}

We report here a large set of double-star measurements made at the $4.1 \mathrm{~m}$ Southern Astrophysical Research Telescope (SOAR) with the speckle camera, HRCam. This paper continues the series published by Tokovinin, Mason, \& Hartkopf (2010a, hereafter TMH10), Tokovinin et al. (2010b), Hartkopf et al. (2012), Tokovinin (2012), Tokovinin et al. (2014), Tokovinin et al. (2015, hereafter SOAR14), and Tokovinin et al. (2016a).

The objects were selected mostly among nearby (within $200 \mathrm{pc}$ ) binaries resolved by Hipparcos, continuing our previous effort in this direction that mirrors the effort in recent years at WIYN (Horch et al. 2017) and DCT (Horch et al. 2015) telescopes on the analogous Northern Hipparcos sample. Similar to the Northern program, the main goal is to identify and follow Hipparcos binaries that show relatively fast orbital motion and that would be good candidates for mass determinations in the coming years. It is especially important to obtain orbital data on these systems in advance of final Gaia results, so that the full power of the Gaia parallaxes can be brought to bear on the determination of the mass sum. We also followed the fast orbital motion of close pairs and subsystems discovered previously at SOAR or elsewhere, with the aim of characterizing their orbits. These data are actively used for orbit calculation (Gomez et al. 2016; Tokovinin 2017; Mendez et al. 2017; Tokovinin 2018b. c; Mason et al. 2018). To provide additional bright targets

Electronic address: atokovinin@ctio.noao.edu

Electronic address: brian.d.mason@navy.mil

Electronic address: rmendez@u.uchile.cl

* Adjunct Astronomer, Lowell Observatory

Electronic address: horche2@southernct.edu when observing conditions are mediocre or poor, we observed as a "filler" potentially interesting neglected pairs suggested by R. Gould (private communication) and binaries with known orbits.

\section{OBSERVATIONS}

\subsection{Instrument}

The observations reported here were obtained with the high-resolution camera (HRCam) - a fast imager designed to work at the $4.1 \mathrm{~m}$ SOAR telescope (Tokovinin \& Cantarutti 2008; Tokovinin 2018a). For practical reasons, the camera was mounted on the SOAR Adaptive Module (SAM, Tokovinin et al. 2016a). However, the laser guide star of SAM was not used (except in 2016 January) because it was not needed and, moreover, reduced the productivity by adding an overhead. The deformable mirror of SAM was passively flattened and the images are seeing-limited. The SAM module contains the atmospheric dispersion corrector (ADC) and helps to calibrate the pixel scale and orientation of HRCam (see SOAR14). The transmission curves of HRCam filters are given in the instrument manual. ${ }^{1}$ We used mostly the Strömgren $y$ filter $(543 / 22 \mathrm{~nm})$ and the near-infrared $I$ filter $(788 / 132 \mathrm{~nm})$.

In 2016 May, at the end of the run, the Luca-DL detector of the HRCam failed after ten years of faithful service and one repair by the vendor (Andor) during this period. In 2016 December, we used the Luca-R camera loaned by the STELES instrument team. With a $75-\mathrm{mm}$ camera lens, the pixel scale was 14.30 mas. A similar Luca-R camera was also used in 2014, as described in SOAR14. However, that frame-transfer CCD had imper-

1 http://www.ctio.noao.edu/soar/sites/default/files/SAM/discretiona 


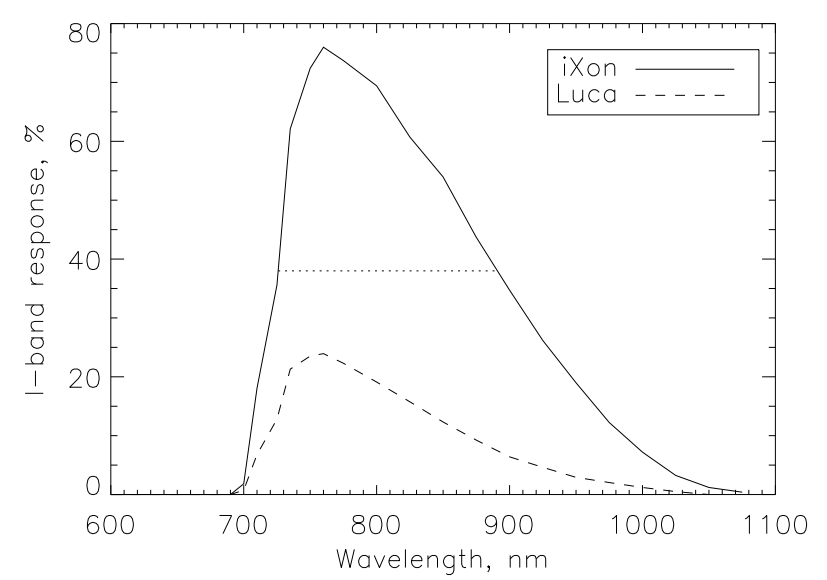

Figure 1. Product of the $I$-filter transmission curve and the detector quantum efficiency. The dotted horizontal line shows the FWHM for the iXon camera.

fect charge transfer in the column direction, leading to a partial loss of resolution. This time, the loaned Luca-R camera was characterized in this respect, and we found that it presents a similar problem, although to a smaller extent. A typical charge spread along the columns was found to be from 2 to 3 pixels. We did not account for this effect in the data processing (Section 2.4), as in 2014 (Tokovinin et al. 2014), but in some cases could reduce its influence by using a reference spectrum with a comparable smear. One consequence of this problem is the reduced resolution in the vertical (usually North-South) direction for the fainter targets.

Meanwhile, Dr. N. Law from the University of North Carolina has kindly loaned us a better electron multiplication (EM) CCD camera, iXon X3 888 (hereafter iXon888), also manufactured by Andor. ${ }^{2}$ Unlike Luca, this is a back-illuminated EM CCD with a substantially higher quantum efficiency and a deeper cooling. The detector has $1024^{2}$ pixels. Sending this export-controlled camera to Chile took longer than expected, so it could be used only in 2017. The larger 13-micron pixel size required a change of the re-imaging lens in HRCam to one with $125 \mathrm{~mm}$ focal length, resulting in the pixel scale of 15.75 mas. The mechanical structure was reinforced to hold this heavier camera. The HRCam PC computer was also replaced. As the cable connecting camera to the computer is short, the PC was located close to the HRCam. The data acquisition software was adapted to the new detector by R. Cantarutti. The minimum exposure time for the standard $200 \times 200$ pixel region was $24 \mathrm{~ms}$, and this exposure time was used mostly throughout 2017. With this exposure time, the $50-\mathrm{Hz}$ vibrations, when present, affect both resolution and sensitivity of HRCam (Tokovinin et al. 2010b; Tokovinin 2018a). A shorter exposure of 6.7 mas is possible in the so-called cropped sensor mode. This mode was tested at the telescope, but not used because switching between readout modes cannot be done rapidly.

Figure 1 shows the quantum efficiency (QE) of the detector multiplied by the transmission of the filter $I$. This filter cuts off only short wavelengths, so the bandwidth depends on the detector's response. The curve for iXon-888 has a Full Width at Half Maximum (FWHM)

\footnotetext{
2 http://www.andor.com/cameras/ixon-emccd-camera-series
}

of $170 \mathrm{~nm}$ (from $725 \mathrm{~nm}$ to $895 \mathrm{~nm}$ ), with the effective wavelength of $824 \mathrm{~nm}$. With such wide bandwidth, the effective wavelength depends on the source color; it is substantially longer for red stars. For comparison, the corresponding QE curve of the Luca-DL camera with the same filter is also plotted; it has the central wavelength of $788 \mathrm{~nm}$ and a narrower FWHM bandwidth of $132 \mathrm{~nm}$.

\subsection{Observing procedure}

In speckle interferometry, the data accumulation takes only a short time (typically $8 \mathrm{~s}$ ). Therefore, the observing efficiency is mainly determined by the telescope slew and setup (centering of the star, setting the ADC, changing filters). The standard procedure at SOAR requires the operator to select target coordinates from a list and to command the slew to the new position. When the observing list contains several hundred targets, this is a labor-intensive task.

To address this problem, we developed in 2014 the speckle observing tool, written in IDL. In preparation for the run, targets are selected from the database including all observed objects; it contains the equatorial coordinates, proper motions, magnitudes, separations, date of the last observation at SOAR, and comments. The selected information is used by the observing tool with a graphic user interface. It displays an area of the sky around the selected target in the horizontal (azimuth and elevation) coordinates. The next target is selected by clicking on the display or entering its number. All previous observations of the selected object at SOAR can be listed on the screen, if desired. By pressing a button, the target coordinates for the current moment (accounting for the proper motion) are sent to the SOAR telescope control system (TCS). The new SOAR TCS moves the telescope if the requested slew is less than $5^{\circ}$ (recently extended to $15^{\circ}$ ), otherwise confirmation of slew by the telescope operator is needed. This tool greatly reduces the load on the telescope operators. At the same time, the target name is sent to the instrument software, hence there is no need to type it. The observer only has to center the target and to select the filter and the detector parameters. Use of this observing tool has improved the efficiency to the point where 300 targets could be observed in one winter night.

The choice of the next target remains manual, considering priorities and variable observing conditions. For example, useful measures of bright stars can be made through transparent clouds or under very poor seeing. So, the observing program contains extra "filler" targets for such situations. The observing tool also helps to optimize telescope slews. Combination of priorities, diverse observing conditions, slew and visibility constraints defines which program stars are actually observed in each run. In 2017, most observations were made remotely from La Serena. This is very convenient, especially for short observing runs.

\subsection{Observing runs}

The observing time for this program was allocated through NOAO (2.5 nights in 2016, programs 16A-0005 and 16B-0044, PI A.T.) and by the Chilean National Time Allocation Committee, CNTAC (4 nights in 2016A, program CN2016AB-4, PI R.A.M.). Some data reported 
Table 1

Observing runs

\begin{tabular}{llrccc}
\hline $\begin{array}{l}\text { Run } \\
\mathrm{H}\end{array}$ & Dates & $\begin{array}{c}\theta_{0} \\
(\mathrm{deg})\end{array}$ & $\begin{array}{c}\text { Pixel } \\
(\mathrm{mas})\end{array}$ & $N_{\text {obj }}$ & $\begin{array}{c}\beta \\
\left({ }^{\prime \prime}\right)\end{array}$ \\
\hline 1 & 2016 Jan 16-18 & -3.00 & 15.23 & 270 & 0.77 \\
2 & 2016 Feb 18-20 & -2.72 & 15.23 & 474 & 0.83 \\
3 & 2016 May 20-23 & 0.20 & 15.23 & 315 & 1.15 \\
4 & 2016 Dec 12 & -12.0 & 14.30 & 41 & 0.64 \\
5 & 2016 Dec 15-17 & 0.30 & 14.30 & 493 & 0.67 \\
6 & 2017 Apr 13 & -0.1 & 15.75 & 152 & 0.82 \\
7 & 2017 May 15 & -0.1 & 15.75 & 201 & 0.80 \\
8 & 2017 Jun 6 & -0.1 & 15.75 & 319 & 0.68 \\
9 & 2017 Jul 14 & -0.1 & 15.75 & 161 & 0.88 \\
10 & 2017 Aug 7 & 0.1 & 15.75 & 116 & 1.02 \\
11 & 2017 Sep 5 & 0.0 & 15.75 & 275 & 0.73 \\
12 & 2017 Oct 4 & -0.15 & 15.75 & 41 & 0.90 \\
13 & 2017 Oct 28 & 0.2 & 15.75 & 122 & 0.55 \\
\hline
\end{tabular}

here (e.g. on calibration binaries) were also collected during observations with HRCam for other programs (2.5 nights in 2015B, 15B-0268, PI C. Briceño, 1 night in 2016A/B, PI B. Pantoja, and 2 nights in 2016A, PI Ji Wang, Kepler-2 follow-up). Measures and discoveries resulting from the Kepler-2 (K2) program are included in this paper. In 2017, two nights per semester were assigned through NOAO for multiple-star observations (PI A.T., programs 17A-0008 and 17B-0066). All observations were made by A.T., sharing the allocated time between programs to cover the whole sky and to improve temporal cadence for pairs with fast orbital motion.

Table 1 lists the observing runs, the calibration parameters (position angle offset $\theta_{0}$ and pixel scale in mas), and the number of objects observed for all programs covered in each run. Its last column gives the median FWHM of the re-centered images, $\beta$, determined during data processing.

Run 1 (2.5 nights in 2016 January) was dedicated to observations of young stars (PI C. Briceño) in Orion and Chamaeleon, see Briceño \& Tokovinin (2017). The image quality (hence sensitivity) was improved using the UV laser, allowing us to observe stars of $I \sim 13$ mag with exposure times of 0.1 or $0.2 \mathrm{~s}$ (not quite at the diffraction limit, though, with a median FWHM of $00^{\prime \prime} 33$ ). When the main targets were not visible, double stars were observed without laser in the standard speckle mode. The seeing was good during most of this run.

Run 2 (2.5 nights in 2016 February) was split between observations of multiple stars (0.5 nights), Hipparcos binaries (2 nights), and the program of B. Pantoja (0.5 night). It enjoyed clear skies and slow wind speed, with average seeing. On the last night of the run, the phenomenon of optical ghosts was observed, as described below in Section 2.6.

Run 3 (4 nights in 2016 May) suffered from poor weather and technical problems. The first night started with a strong wind and a high humidity of $83 \%$. The seeing was extremely poor $\left(2^{\prime \prime}\right.$ to $\left.3^{\prime \prime}\right)$. The telescope was closed for high humidity most of the night, so only 44 bright stars could be observed. The following night was clear, with a strong wind, poor seeing, and occasional passage of transparent clouds. The third night was lost to clouds. When some bright stars were observed between the clouds, frequent failures of the LucaDL camera prevented operation. On the last night of the run (also mostly cloudy) we replaced the camera, but these data are discarded here, being insufficient in both quality and quantity for a meaningful analysis. The first two nights of this run were assigned to the Kepler-2 (K2) follow-up, which is the Yale program managed by Ji Wang (Caltech). He provided the list of targets including all known binaries from the Washington Double Star Catalog, WDS (Mason et al. 2001) in the K2 fields (RA range from $16 \mathrm{~h}$ to $20 \mathrm{~h}$, declination from $-31^{\circ}$ to $\left.-25^{\circ}\right)$. Pairs wider than $4^{\prime \prime}$ could not be measured with HRCam; however, we discovered 10 new components in known binaries (see Section 3.3). The two last nights (May 22 and 23) were assigned to the program of R. Mendez (CNTAC) on Hipparcos binaries. In this run, the new SOAR TCS was operational, allowing small slews to be commanded directly by the speckle observing tool.

Run 5 (2.5 nights in 2016 December) was preceded by 2 hours of engineering observations on December 12 , treated here as a separate run 4. As described in Section 2.1. we used the loaned Luca-R camera in these runs. The sky was clear, the seeing was average.

Run 6 in 2017 April used for the first time the new iXon-888 CCD camera, on an engineering night with a strong wind and occasional transparent clouds.

Run 7 in 2017 May used one allocated night. For several hours the telescope remained closed owing to high humidity.

Run 8 on 2017 June 6 used one full night allocated for the multiple-star program. The sky was mostly clear. A record number of 319 targets were observed during this night.

Run 9 in 2017 July was a half-night allocation for the program by B. Pantoja. Some observations were made through transparent clouds.

Run 10 used the engineering time on a partially cloudy night, when other planned tasks could not be accomplished.

Run 11 was almost a full night of 2017 September 5 (engineering). The regular allocated night of September 11 was lost to clouds.

Run 12 on 2017 October 4 used only 2 hours of engineering time, again observing through the clouds.

Run 13 on 2017 October 28 (half-night) enjoyed a clear sky and good seeing. The increased sensitivity of the new camera allowed us to observe some stars as faint as $I=14 \mathrm{mag}$ without adaptive correction. However, SOAR vibrations affected some data of this run.

\subsection{Data processing}

Data processing is described in TMH10 and subsequent papers of this series. We recall it here briefly, emphasizing the caveats. A series of short-exposure images are recorded as FITS cubes, typically of $200 \times 200 \times 400$ pixels size, two cubes per target and per filter. A larger image size of $400 \times 400$ pixels is used for pairs wider than $1^{\prime \prime} 5$. The power spectrum (PS) of each data cube is computed after subtracting the bias and applying a threshold to eliminate noise in empty pixels. In the case of iXon888 cooled to $-60^{\circ} \mathrm{C}$, the dark current is negligible, while the bias has a gradient in the vertical direction only; the software was adapted accordingly. Along with the PS, the program computes the average re-centered image and the shift-and-add (SAA) image centered on the brightest pixel. The auto-correlation function $(\mathrm{ACF})$ is computed later from the PS, filtered to remove low spatial frequen- 
cies.

Binary companions are detected in the ACF. Their parameters (position angle $\theta$, separation $\rho$ and magnitude difference $\Delta m$ ) and their formal errors are determined by fitting a model to the high-frequency part of the PS; the model is a product of the reference PS and the PS of two point sources. In most cases, the reference is derived from the azimuthally averaged PS of the object itself (TMH10). Vibrations, telescope wind shake, and residual aberrations such as astigmatism create twodimensional patterns in the PS that are not captured by the model. Using another observed object (either unresolved or with a substantial magnitude difference) helps here, as explained in Tokovinin et al. (2016a). However, the PS structure constantly evolves in time, complicating its modeling.

Measurements of binaries wider than $\sim 0$ ". 1 are not sensitive to the PS model and are very robust. In contrast, for close pairs with a substantial magnitude difference $(\Delta m)$, the results of the fitting procedure do depend on the PS structure, the use (or not) of the real on-sky reference, and its conformity to the actual PS. Some measures presented here are affected by these poorly quantified biases. Differences between positions measured in the $y$ and $I$ filters are indicative of such cases. Measures of binaries at or below the diffraction limit (27 mas in the $y$ filter and 40 mas in the $I$ filter) should also be treated with caution. Less reliable measurements are marked by colons.

Yet another caveat is related to the differential photometry of binaries wider than $\sim 1^{\prime \prime}$. The speckle signal is reduced by anisoplanatism, biasing the derived $\Delta m$ to larger values. If the pair is resolved in the centered images, an alternative photometric procedure corrects for this bias (see TMH10). The resolution in the centered images, $\beta$, is also determined in the process. However, images of wide pairs can be partially truncated, especially when the wind-induced telescope shake causes substantial image wander or when the seeing is particularly poor. Such situations also lead to an over-estimate of $\Delta m$. It is safe to consider the published $\Delta m$ of wide pairs as upper limits. The photometry is reliable when there are several mutually agreeing measurements of $\Delta m$.

Speckle processing determines the PA of the pair modulo $180^{\circ}$. When the companion is seen in the SAA images, the correct quadrant can be chosen, provided that $\Delta m>0.3 \mathrm{mag}$; otherwise, the two peaks in the SAA image are equal and the strongest one cannot be identified. Quadrants defined in this way are marked by the flag "q" in the data tables. The flag "** indicates binaries resolved in the centered images, where the quadrants are also known.

\subsection{Calibration of position angle and scale}

The calibration of the PA and pixel scale was done with respect to 64 wide pairs, as explained in SOAR14. It was revisited and improved here by including more calibrators and the latest data. Moreover, the motion of some calibrators is now modeled by orbits adjusted to fit the SOAR data, rather than by linear functions of time. A few stars showing obvious deviations from the models were removed from the list of calibrators. One such ex-calibrator that turned out to be a triple system is presented below in Section 3.4. A typical rms deviation

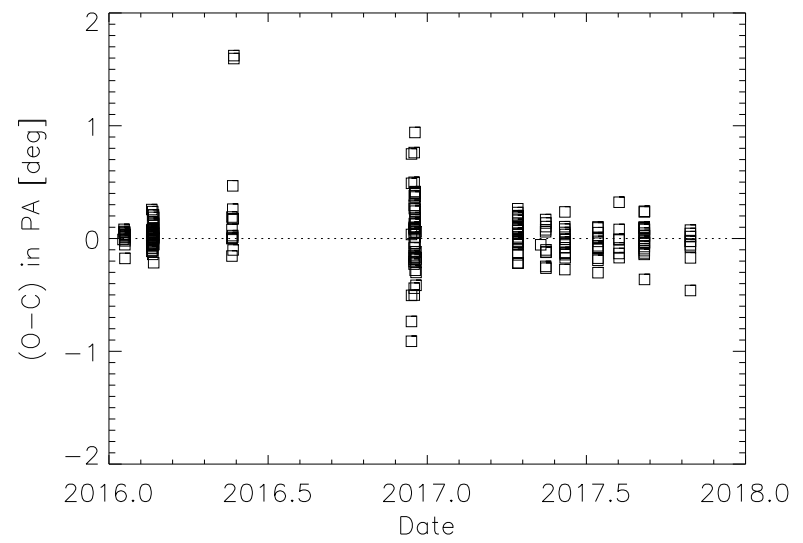

Figure 2. Residual in position angle of the calibration stars, plotted vs. time.

of the calibrator binaries from the models is from 1 to 3 mas in both radial and tangential directions. Overall, measures of the calibrators comprise about $10 \%$ of all measures presented here.

In run 3, the new SOAR TCS was used for the first time. We found that the instrument PA, nominally set at $0^{\circ}, 90^{\circ}$, or some other round number, was incorrect in a small number of cases, causing manifestly wrong angle measurements. This prompted us to recompute all instrument PAs using the information on the telescope elevation, Nasmyth rotator angle, and star position. The recomputed angles differed from their nominal round values; they were used in the data reduction. With the recomputed instrument angles, the rms scatter in the PA of the calibration binaries decreased from 0.7 to 0.4 , indicating the appropriateness of this correction. However, the remaining scatter is still substantially larger than normal. It is possible that the mechanical rotation of the Nasmyth bearing had failures preventing it from reaching the required angle. This problem apparently persisted in run 5, but the PAs were not recomputed because this did not reduce the scatter of the calibrators, 0.4 . The small engineering run 4 suffered from the communication problem between the instrument software and the TCS; some PAs in these data might be erroneous. In contrast, the calibrator observations in 2017 show a small PA scatter from 0.1 to 0.2 in all runs.

Figure 2 plots the residual deviations of the calibrators in PA vs. time. The distribution of points in time corresponds to the observing runs in Table 11. Despite the correction of the instrument angles in run 4, there is one deviant measure. The run 5 also shows a larger than usual scatter in the PA residuals of the calibrators. When all 231 observations of the calibrators during two years are treated as one data set, the rms scatter in PA is 0.25 . The global rms scatter of the scale factor is 0.0041 .

Four binaries (WDS J04136+0743, J07277+2127, J09285+0903, and J22409+1433) have very accurate orbits based on long-baseline interferometry (Muterspaugh et al. 2010a, b). A total of 10 measures of those binaries have mean residual in PA of -0.15 with rms scatter of 0.28 . The mean residual in separation is 3.3 mas with the rms of 2.0 mas. This comparison is the external check of the data accuracy. The majority of visual orbits are less accurate than our measures.

\subsection{Optical ghosts}


During the first hours of the 2016 February 20 night, a phenomenon of optical ghosts (OG) was observed. The sky was clear, the wind speed was low or zero. We pointed and unexpectedly resolved ADS 3701, a known "ghost" binary (Tokovinin 2012). However, a bright star HR 1585 observed immediately after also displayed a similar doubling. Both bright objects were observed with short 2-ms exposures, eliminating potential effect of telescope vibration. Figure 3 shows the speckle ACFs of these two objects in three filters. Unlike real double stars, the separation of the "companions" increases in proportion to the wavelength. Moreover, we see the second, fainter companions with double separation. The phenomenon is obviously associated with diffraction on a periodic structure where the first and second diffraction orders are seen. The separation of the first-order diffraction maximum $\rho \sim 00^{\prime \prime} 1$ is related to the grating period $b=\lambda / \rho$, with $b$ calculated to be from 0.8 to $1.1 \mathrm{~m}$.

The OGs were observed in three objects located close to each other on the sky, then disappeared in the following group of objects in the same sky area, and reappeared again in two more episodes, each counting several successive objects. The three OG episodes occurred during a time period of about one hour (from UT 0:46 to 1:37) and no more OGs were seen for the rest of the night. Data examination revealed that similar OGs occurred on the previous night around UT 0:18 and 9:26. The wind speed on the previous night was also very low.

Table 2 provides the circumstances of some OG observations that might help in finding their origin. The first three columns give the UT date of the observation, filter, and WDS designation of the target. The WDS is used only for convenience, as OGs are unrelated to binary companions (in fact, $04357+0127$ is the single star HIP 21411). Then follow the angle of the Nasmyth rotator ROT, the telescope azimuth AZ, and the elevation EL. The difference EL-ROT shows the instrument angle relative to the telescope primary mirror or, equivalently, the parallactic angle. The last two columns give the position angle $\theta$ and the separation $\rho$ of the OGs measured as if they were double stars.

The position angles of OGs observed on both nights are confined within a narrow range, close to but not exactly aligned with the North-South direction. The parallactic angle ranged over $32^{\circ}$, so OGs are not aligned in the vertical direction. The OGs were observed when the telescope was pointing to the North-North-East, at moderate elevation. They appeared and disappeared on a time scale of several minutes; however, in the pair of data cubes of the same star taken one after another the OGs are always similar.

The nature of OGs is mysterious. We could reproduce the OGs in simulated speckle images by placing in the beam a fixed periodic phase screen, in addition to the random atmospheric perturbation corresponding to the 0.7 seeing. By trial and error we found that the clipped sine wave (only positive half-periods, zero otherwise) with a spatial period $b$ of $1 \mathrm{~m}$ and a path-length amplitude of $0.2 \mu \mathrm{m}$ matches the OGs seen in various filters, creating two diffraction orders of approximately correct relative intensity.

The origin of such quasi-periodic phase disturbances is not known. They are certainly not related to the instrument or telescope, as evidenced by the position an-
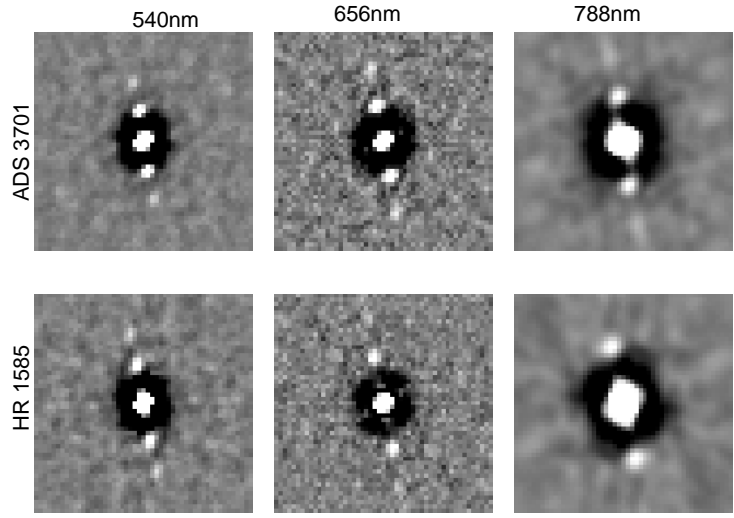

Figure 3. ACFs of ADS 3701 (top row) and HR 1585 (bottom row) in three filters showing OGs. Fragments of ACFs of $51 \times 51$ pixels $\left(00^{\prime \prime} 78\right)$ recorded on 2016 February 20 are shown. North is up, East to the right, the intensity scale is arbitrary.

gles. The fact that OGs appear only episodically under zero-wind conditions suggests that they might be a transient atmospheric phenomenon like stratification or waves. Systematic wavefront distortion under low wind has been observed at the VLT by Sauvage et al. (2015). OGs corresponding to the grating period of $\sim 2 \mathrm{~m}$ were seen during previous speckle runs at SOAR (see Fig. 11 in TMH10). However, OGs with two diffraction orders and a larger separation were observed at SOAR for the first time only now. The OG phenomenon can explain some false double-star discoveries made with speckle interferometry in the past. We mistakenly assumed HIP 75050 to be a newly resolved pair before realizing that its measure refers to the OG.

\section{RESULTS}

\subsection{Data tables}

We do not report here the results belonging to other PIs, namely the Orion and Chamaeleon (Briceño \& Tokovinin 2017) surveys and the targets observed for B. Pantoja. However, observations of binaries in the K2 fields (PI Ji Wang) are published here with the PI's permission.

The data tables have the same format as in the previous papers of this series. They are available in full only electronically. Table 3 lists 2483 measures of 1570 resolved pairs and subsystems, including 44 newly resolved pairs. The columns of Table 3 contain (1) the WDS (Mason et al. 2001) designation, (2) the "discoverer designation" as adopted in WDS, (3) an alternative name, mostly from the Hipparcos catalog, (4) Julian year of observation, (5) filter, (6) number of averaged individual data cubes, $(7,8)$ position angle $\theta$ in degrees and internal measurement error in tangential direction $\rho \sigma_{\theta}$ in mas, $(9,10)$ separation $\rho$ in arcseconds and its internal error $\sigma_{\rho}$ in mas, and (11) magnitude difference $\Delta m$. An asterisk follows if $\Delta m$ and the true quadrant are determined from the resolved re-centered image; a colon indicates that the data are noisy and $\Delta m$ is likely overestimated (see TMH10 for details); the flag "q" means that the quadrant is determined from the SAA image (Section 2.4). Note that in the cases of multiple stars, the positions and photometry refer to the pairings between individual stars, not the photo-centers of subsystems.

For binary stars with known orbital elements, columns (12-14) of Table 3 list the residuals to the ephemeris 
Table 2

Examples of optical ghosts

\begin{tabular}{|c|c|c|c|c|c|c|c|c|}
\hline Date (UT) & Filt. & $\begin{array}{c}\text { WDS } \\
\alpha, \delta(2000)\end{array}$ & $\begin{array}{c}\mathrm{ROT} \\
\left({ }^{\circ}\right)\end{array}$ & $\begin{array}{l}\mathrm{AZ} \\
\left({ }^{\circ}\right)\end{array}$ & $\begin{array}{l}\mathrm{EL} \\
\left({ }^{\circ}\right)\end{array}$ & $\begin{array}{c}\mathrm{ROT}_{\left({ }^{\circ}\right)}-\mathrm{EL} \\
\end{array}$ & $\begin{array}{c}\theta \\
\left({ }^{\circ}\right)\end{array}$ & $\begin{array}{c}\rho \\
\left({ }^{\prime \prime}\right)\end{array}$ \\
\hline 2016-02-20 00:18 & $\mathrm{I}$ & $04357+0127$ & 77.3 & 334.6 & 55.3 & 22.0 & 161.7 & 0.178 \\
\hline $2016-02-2009: 26$ & I & $14375+0217$ & 58.8 & 358.4 & 57.3 & 1.5 & 167.6 & 0.231 \\
\hline 2016-02-20 09:26 & $\mathrm{y}$ & $14375+0217$ & 58.8 & 358.4 & 57.3 & 1.5 & 166.2 & 0.153 \\
\hline 2016-02-21 01:12 & $\mathrm{y}$ & $05348+0929$ & 65.9 & 339.6 & 47.9 & 18.1 & 167.5 & 0.155 \\
\hline 2016-02-21 01:14 & $\mathrm{H} \alpha$ & $05348+0929$ & 65.9 & 339.6 & 47.9 & 18.1 & 167.0 & 0.184 \\
\hline 2016-02-21 01:16 & $\mathrm{y}$ & $05079+0830$ & 73.0 & 328.8 & 45.9 & 27.1 & 164.9 & 0.092 \\
\hline 2016-02-21 01:30 & $\mathrm{y}$ & $05354-0555$ & 93.0 & 321.4 & 60.0 & 33.1 & 164.9 & 0.187 \\
\hline
\end{tabular}

Table 3

Measurements of double stars at SOAR (fragment)

\begin{tabular}{|c|c|c|c|c|c|c|c|c|c|c|c|c|c|}
\hline $\begin{array}{c}\text { WDS } \\
(2000)\end{array}$ & $\begin{array}{c}\text { Discoverer } \\
\text { Designation }\end{array}$ & $\begin{array}{l}\text { Other } \\
\text { name }\end{array}$ & $\begin{array}{l}\text { Epoch } \\
+2000\end{array}$ & Filt & $\mathrm{N}$ & $\begin{array}{c}\theta \\
(\operatorname{deg})\end{array}$ & $\begin{array}{c}\rho \sigma_{\theta} \\
(\mathrm{mas})\end{array}$ & $\begin{array}{c}\rho \\
(\prime \prime)\end{array}$ & $\begin{array}{c}\sigma \rho \\
(\mathrm{mas})\end{array}$ & $\begin{array}{c}\Delta m \\
(\mathrm{mag})\end{array}$ & $\begin{array}{c}\mathrm{O}-\mathrm{C}]_{\theta} \\
(\mathrm{deg})\end{array}$ & $\begin{array}{c}\mathrm{O}-\mathrm{C}]_{\rho} \\
\left({ }^{\prime \prime}\right)\end{array}$ & $\begin{array}{c}\text { Reference } \\
\text { code* }^{*}\end{array}$ \\
\hline $00024+1047$ & A $1249 \mathrm{AB}$ & HIP 190 & 17.6802 & I & 2 & 246.4 & 0.5 & 0.3003 & 0.1 & $0.8 \mathrm{q}$ & -0.1 & 0.041 & Zir2003 \\
\hline $00029-7436$ & TDS 3 AB & $\mathrm{CD}-751309$ & 17.6804 & I & 2 & 46.8 & 1.4 & 1.5189 & 0.5 & $0.6 *$ & & & \\
\hline \multirow[t]{2}{*}{$00036-3106$} & TOK 686 & HIP 290 & 16.9487 & $\mathrm{I}$ & 3 & 13.3 & 0.8 & 0.1293 & 12.0 & 3.6 & & & \\
\hline & & & 17.6801 & $\mathrm{I}$ & 2 & 24.1 & 10.0 & 0.1320 & 1.0 & 4.1 & & & \\
\hline $00039-5750$ & I 700 & HIP 306 & 16.9596 & $\mathrm{I}$ & 2 & 167.1 & 0.3 & 0.2557 & 0.1 & 0.3 & & & \\
\hline $00061+0943$ & HDS 7 & HIP 510 & 17.6802 & I & 2 & 196.3 & 0.3 & 0.2604 & 0.1 & 0.3 & 0.2 & 0.003 & FMR2017c \\
\hline $00098-3347$ & SEE 3 & HIP 794 & 17.6801 & I & 2 & 123.6 & 0.1 & 0.9069 & 0.1 & $1.0 *$ & 1.9 & 0.007 & Hrt2010a \\
\hline $00100+0835$ & A 1801 & HIP 807 & 17.6802 & I & 2 & 198.5 & 0.1 & 0.3164 & 0.2 & 0.3 & & & \\
\hline $00106-7313$ & I $43 \mathrm{AB}$ & HIP 865 & 17.6011 & I & 3 & 196.0 & 0.7 & 0.4956 & 0.7 & 1.3 & -0.6 & -0.009 & Cve2010e \\
\hline \multirow[t]{2}{*}{$00121-5832$} & RST 4739 & HIP 975 & 16.9596 & I & 2 & 282.9 & 0.2 & 0.2853 & 0.1 & 0.2 & & & \\
\hline & & & 17.6008 & I & 2 & 279.3 & 1.3 & 0.2776 & 0.5 & 0.0 : & & & \\
\hline $00135-3650$ & HDS 32 & HIP 1083 & 16.9487 & I & 2 & 11.4 & 0.5 & 0.2592 & 0.2 & $0.8 \mathrm{q}$ & 0.0 & 0.000 & Tok2017b \\
\hline
\end{tabular}

* References to VB6 are provided at/http://ad.usno.navy.mil/wds/orb6/wdsref.txt

Table 4

Unresolved stars (fragment)

\begin{tabular}{|c|c|c|c|c|c|c|c|c|c|}
\hline $\begin{array}{l}\text { WDS (2000) } \\
\alpha, \delta(\mathrm{J} 2000)\end{array}$ & $\begin{array}{c}\text { Discoverer } \\
\text { Designation } \\
\text { or other name }\end{array}$ & $\begin{array}{c}\text { Hipparcos } \\
\text { or other } \\
\text { name }\end{array}$ & $\begin{array}{l}\text { Epoch } \\
+2000\end{array}$ & Filter & $\mathrm{N}$ & $\begin{array}{c}\rho_{\min } \\
(\operatorname{arcsec})\end{array}$ & $\begin{array}{c}5 \sigma \text { Detecti } \\
\Delta m\left(0^{\prime \prime} 15\right) \\
(\mathrm{mag})\end{array}$ & $\begin{array}{c}{ }_{0} \text { Limit } \\
\Delta m\left(1^{\prime \prime}\right) \\
(\mathrm{mag})\end{array}$ & $\begin{array}{l}\Delta m \\
\text { flag }\end{array}$ \\
\hline $00219-2300$ & RST $5493 \mathrm{~A}$ & HIP 1732 & 17.6801 & I & 2 & 0.041 & 2.53 & 5.68 & \\
\hline $00291-0742$ & MLR 2 & HIP 2275 & 17.6801 & I & 2 & 0.041 & 2.66 & 4.41 & \\
\hline 00313-1909 & B 6 & HD 2797 & 17.6801 & I & 2 & 0.041 & 3.10 & 4.82 & \\
\hline $00324+0657$ & $\mathrm{MCA} 1 \mathrm{Aa}, \mathrm{Ab}$ & HIP 2548 & 16.9595 & I & 2 & 0.040 & 2.93 & 4.61 & \\
\hline & & & 16.9595 & $\mathrm{H} \alpha$ & 2 & 0.041 & 3.32 & 4.24 & \\
\hline & & & 17.6802 & I & 2 & 0.041 & 2.22 & 4.56 & \\
\hline & & & 17.6802 & $\mathrm{y}$ & 1 & 0.028 & 3.53 & 5.79 & \\
\hline & & & 17.6802 & $\mathrm{H} \alpha$ & 1 & 0.039 & 3.73 & 5.01 & \\
\hline $00366-4908$ & HIP 2888 & HIP 2888 & 16.9596 & I & 2 & 0.040 & 4.13 & 6.21 & \\
\hline $00374-3717$ & I 705 & HIP 2944 & 16.9596 & I & 2 & 0.040 & 4.07 & 6.29 & \\
\hline & & & 16.9596 & $\mathrm{y}$ & 2 & 0.028 & 4.39 & 6.05 & \\
\hline $00467-0426$ & $\mathrm{LSC} 10 \mathrm{Aa}, \mathrm{Ab}$ & HIP 3645 & 16.9569 & I & 2 & 0.040 & 3.14 & 5.10 & \\
\hline
\end{tabular}

position and code of reference to the orbit adopted in the Sixth Catalog of Orbits of Visual Binary Stars (Hartkopf, Mason \& Worley 2001, hereafter VB6). ${ }^{3}$

Table 4 contains the data on 426 unresolved stars, some of which are listed as binaries in WDS or resolved here in other filters. Columns (1) through (6) are the same as in Table 3, although column (2) also includes other names for objects without discoverer designations. For stars that do not have entries in WDS, WDS-style codes based on the J2000 position are listed in column (1). Column (7) is the estimated resolution limit, equal

\footnotetext{
3 See http://ad.usno.navy.mil/wds/orb6/wdsref .html
}

to the diffraction radius $\lambda / D$ for good-quality data and larger for poor data (the effective resolution limit is computed from the maximum spatial frequency where the signal in the power spectrum stands above the noise, see TMH10). Columns $(8,9)$ give the $5 \sigma$ detection limits $\Delta m$ at 0.15 and $1^{\prime \prime}$ separations determined by the procedure described in TMH10. When two or more data cubes are processed, the largest $\Delta m$ value is listed. The last column marks with colons noisy data mostly associated with faint stars. In such cases, the quoted detection limits might be too large (optimistic); however, the information that these stars were observed and no companions were found is still useful. In a few instances, 
$\Delta m\left(0^{\prime \prime} .15\right)=0$ indicates that the automatic procedure failed to determine detection limit at close separation.

\subsection{Most Tycho binaries are spurious}

The targets in run 4 featured all WDS binaries in the Kepler-2 fields, including those discovered by the $T y$ cho mission (discoverer codes TDS and TDT). There are 17 targets with these codes in our data. One of those, TDT 721 , is too wide to be resolved. Of the remaining 16 , only one (TDT 3 at $1^{\prime \prime} .5$ ) is confirmed. The parameters of the Tycho pairs make them easily accessible to HRCam. We therefore conclude that a large number of Tycho binaries are spurious. Their supposed separations range from 0. . 4 to $3^{\prime \prime}$. Interestingly, we have resolved two more pairs in this group, but at different separations: WDS J16086-2540 at $1^{\prime \prime} .89$ and $\Delta I=6.1 \mathrm{mag}$ (the TDS 9771 is listed with 0. . 4 separation and $\Delta m=0.12$ mag) and WDS J17022-2820 at $0 . .63$ and $\Delta I=3.8 \mathrm{mag}$ (TDT 186 is listed at $0 . \prime 4$ and $\Delta m=0.44 \mathrm{mag}$ ). These faint companions are random discoveries unrelated to the previously claimed Tycho pairs.

The WDS contains 14,170 Tycho pairs; 330 of those have a code 'X', i.e. are marked as spurious, while 1201 are confirmed. The veracity of most Tycho pairs still waits for confirmation.

\subsection{Newly resolved pairs}

Table 5 lists 44 newly resolved pairs. Its format is similar to that of Table 3. For some multiple systems, we used existing discoverer codes and simply added new component designations. The last two columns of Table 5 contain the spectral type (as given in SIMBAD) and the Hipparcos parallax $p$ (van Leeuwen 2007). The Gaia (Gaia collaboration 2016) parallaxes, when available, are preferred; they are marked by asterisks. We comment on these objects below. The following abbreviations are used: PM - proper motion, CPM - common proper motion, RV - radial velocity, SB1 and SB2 single- and double-lined spectroscopic binaries. Orbital periods are estimated from projected separation as

$$
P^{*}=(\rho / p)^{3 / 2} M^{-1 / 2},
$$

where $\rho$ is the angular separation (assumed to equal the semimajor axis), $p$ is the parallax, $M$ is the mass sum, and $P^{*}$ is the period in years. Statistical relation of these estimates to true periods is discussed by Tokovinin (2014). Data from the spectroscopic GenevaCopenhagen Survey, GCS (Nordström et al. 2004) are used for some targets.

HIP 20802 (HD 28388) is a G6V astrometric binary (Makarov \& Kaplan 2005), on the California exoplanet search program (Isaacson \& Fischer 2010); Nidever et al. (2002) found an RV trend. Estimated period: 25 years.

HIP 35986 (HD 58249) has not been detected as an SB by the GCS, possibly owing to its fast axial rotation. Its small separation implies $P^{*} \sim 10 \mathrm{yr}$. The pair was not measured in run 5 because of the charge transfer problem, but was resolved again in 2017.3 (run 6) at similar PA and larger separation.

HIP 37012 (HD 45698) is a chemically peculiar Ap star resolved at $0^{\prime \prime} 14$, implying $P^{*}=65$ years.

HIP 41800 (HD 72881) is a chemically peculiar Ap star without any prior hints on its binarity; $P^{*}=30 \mathrm{yr}$.
The 6 measures show a large scatter. However, without considering uncertain measures marked by colons, the position is stable over one year.

HIP 44914 (CD-37 5499) is a high-PM K5V dwarf star with only two references in SIMBAD. The new faint component at $1^{\prime \prime} .34$ remained fixed in one year, hence it is physical. The period is long, $P^{*}=900 \mathrm{yr}$.

HIP 52792 (HD 93527) has a CPM companion HIP 52793 at $30^{\prime \prime}$, which is itself an SB2 according to the GCS. The new close pair Aa,Ab is fast $\left(P^{*}=2 \mathrm{yr}\right)$, and we indeed see its fast retrograde motion in one year.

HIP 53938 (HD 95699, V360 Vel) is a chemically peculiar Ap star with periodic flux variability. The separation has slightly increased in one year, matching $P^{*}=55 \mathrm{yr}$. There is a physical companion at 19.2 .

HIP $54553(B D+16$ 2222) is a nearby M0V dwarf GJ 9348 with a relatively fast PM. The new companion at 2.6 is confirmed as physical in 2018.

$H I P 57600$ (HD 102698) is another nearby $\mathrm{K} 4 \mathrm{~V}$ dwarf known as an astrometric (acceleration) binary (Makarov \& Kaplan 2005). A fast retrograde motion of the new pair is seen, in agreement with $P^{*}=5 \mathrm{yr}$. The wide companion TOK 281 with similar PM listed in WDS at $407^{\prime \prime}$ separation is likely optical.

HIP 57698 (HD 102804) is on the Magellan exoplanet search program. The newly found companion at 0 ".6 60 can cause a measurable RV trend despite its long $P^{*}=350$ yr. The pair is confirmed as physical by its re-observation in 2017.

HIP $58084(B D-13$ 3470) is a $\mathrm{K} 6 \mathrm{~V}$ dwarf found here to be a 0. . 07 pair with nearly equal components and fast retrograde motion, in agreement with the estimated period $P^{*}=10$ yr.

HIP 62445 (HD 111170, V940 Cen) is a young chromospherically active G9IV star in the Sco-Cen association. The newly resolved pair has $P^{*}=5 \mathrm{yr}$ and shows some orbital motion in one year. The large discrepancy between the Hipparcos (7.7 mas) and Gaia (13.2 mas) parallaxes is presumably caused by the effect of orbital motion on the astrometric data reduction.

HIP 62985 ( $\psi$ Vir, HR 4902) is a bright M-type giant with 149 references in SIMBAD. The faint binary companion detected in 1975 by lunar occultations (OCC 387 in the WDS) was directly resolved for the first time in 2016 at $0^{\prime \prime} .21$ and confirmed next year. The estimated period is $\sim 100$ years.

HIP 63012 (HD 112145) is featured in the GCS, but otherwise it has attracted no interest so far. The new $0^{\prime \prime}$.16 pair with $P^{*}=80 \mathrm{yr}$ is also not interesting because of its slow expected motion.

HIP 6337r (HD 112636) has a companion at 0.42. The resolution is very secure, but no second measure has been taken so far. Only a slow motion is expected, $P^{*}=160 \mathrm{yr}$.

HIP 69113 (HD 123445) is a bright B9V member of the Sco-Cen association. It was pointed instead of the fainter pair DE, at 5".3 from the component A. Detection of the new 56 mas, nearly equal pair $\mathrm{Aa}, \mathrm{Ab}$ is secure; it is confirmed in 2018, at closer separation. The estimated period is $P^{*}=12$ years.

HIP 69549 (HD 124605) has a fast PM and an RV of $-88.9 \mathrm{~km} \mathrm{~s}^{-1}$; it is likely metal-poor. Its resolution at 0 ".08 in 2016.14 is secure; however, the pair closed down 
Table 5

Newly resolved pairs

\begin{tabular}{|c|c|c|c|c|c|c|c|c|c|}
\hline $\begin{array}{l}\text { WDS } \\
(2000)\end{array}$ & $\begin{array}{c}\text { Discoverer } \\
\text { Designation }\end{array}$ & $\begin{array}{l}\text { Other } \\
\text { name }\end{array}$ & $\begin{array}{l}\text { Epoch } \\
+2000\end{array}$ & Filt & $\begin{array}{c}\theta \\
(\mathrm{deg})\end{array}$ & $\begin{array}{c}\rho \\
(I \prime)\end{array}$ & $\begin{array}{c}\Delta m \\
(\mathrm{mag})\end{array}$ & $\begin{array}{l}\text { Spectral } \\
\text { type }\end{array}$ & $\begin{array}{c}p \\
\text { (mas) }\end{array}$ \\
\hline $02460-0457$ & $\mathrm{BU} 83 \mathrm{Aa}, \mathrm{Ab}$ & HIP 12912 & 16.9590 & $\mathrm{I}$ & 67.8 & 0.2231 & 4.3 & F3V & 13.2 \\
\hline $04274-2912$ & TOK 709 & HIP 20802 & 16.1373 & I & 68.7 & 0.1278 & $2.1 \mathrm{q}$ & G6V & $12.1^{*}$ \\
\hline $06272-3706$ & TOK 710 & HIP 30712 & 16.1330 & I & 159.4 & 0.1392 & $2.3 \mathrm{q}$ & ApSrEu & $6.0^{*}$ \\
\hline $07250+0406$ & TOK 711 & HIP 35986 & 16.1346 & I & 156.2 & 0.0301 & 0.7 & G0V & $6.7^{*}$ \\
\hline $08314-6531$ & TOK 712 & HIP 41800 & 16.1373 & I & 203.3 & 0.0517 & $1.1 \mathrm{q}$ & $\mathrm{ApSi}$ & 4.0 \\
\hline 09090-3802 & TOK 713 & HIP 44914 & 16.1375 & I & 266.5 & 1.3401 & $2.0 *$ & $\mathrm{~K} 5 \mathrm{~V}$ & $14.4^{*}$ \\
\hline $10476-1538$ & TOK $714 \mathrm{Aa}, \mathrm{Ab}$ & HIP 52792 & 16.1376 & I & 72.9 & 0.0294 & 0.1 & F7II/III & $13.1^{*}$ \\
\hline $11022-4230$ & TOK $715 \mathrm{Aa}, \mathrm{Ab}$ & HIP 53938 & 16.1376 & I & 125.0 & 0.1174 & $1.7 \mathrm{q}$ & ApSrEuCr & 5.2 \\
\hline $11098+1531$ & TOK 716 & HIP 54553 & 16.1351 & I & 248.0 & 2.6180 & $2.0 *$ & MOV & $23.0^{*}$ \\
\hline $11495-1636$ & TOK $717 \mathrm{Aa}, \mathrm{Ab}$ & HIP 57660 & 16.1351 & I & 81.3 & 0.0682 & $0.5 \mathrm{q}$ & $\mathrm{K} 4 \mathrm{~V}$ & $19.0 *$ \\
\hline $11500-5616$ & TOK 718 & HIP 57698 & 16.1377 & I & 202.1 & 0.6002 & $3.2^{1}$ & F3V & $8.4^{*}$ \\
\hline $11547-1401$ & TOK 719 & HIP 58084 & 16.1351 & I & 130.6 & 0.0732 & $0.3:$ & $\mathrm{K} 6 \mathrm{~V}$ & $13.9^{*}$ \\
\hline $12479-5127$ & TOK 720 & HIP 62445 & 16.1353 & I & 176.1 & 0.0485 & 0.7 & G9IVe & $13.2^{*}$ \\
\hline $12544-0932$ & OCC 387 & HIP 62985 & 16.3890 & $\mathrm{y}$ & 111.7 & 0.2116 & 3.7 & M2III & 6.0 \\
\hline $12547-3930$ & TOK 721 & HIP 63012 & 16.1354 & I & 320.8 & 0.1647 & $2.9 \mathrm{q}$ & F7V & $6.7^{*}$ \\
\hline $12592-6256$ & TOK 722 & HIP 63377 & 16.1351 & I & 55.1 & 0.4176 & $2.4 \mathrm{q}$ & G3V & 11.2 \\
\hline $14089-4328$ & HJ $4653 \mathrm{Aa}, \mathrm{Ab}$ & HIP 69113 & 17.2833 & I & 95.2 & 0.0555 & 0.1 & $\mathrm{~B} 9 \mathrm{~V}$ & 6.2 \\
\hline $14142+1805$ & TOK 723 & HIP 69549 & 16.1382 & I & 350.6 & 0.0816 & 2.4 & G0V & $11.6^{*}$ \\
\hline $14275-3527$ & TOK 724 & HIP 70693 & 16.1354 & I & 138.5 & 0.0532 & 0.2 & F8V & 10.8 \\
\hline $14494-5726$ & HDS 2092 BC & HIP 72492 & 16.1406 & I & 170.0 & 0.0567 & 0.3 & F5V & $8.2^{*}$ \\
\hline $15537-0429$ & TOK 725 & HIP 77843 & 16.1410 & I & 32.8 & 0.1270 & $1.3 \mathrm{q}$ & $\mathrm{F} 8 / \mathrm{G} 0 \mathrm{~V}$ & 12.5 \\
\hline $16012-4632$ & SEE $254 \mathrm{Aa}, \mathrm{Ab}$ & HIP 78485 & 17.4324 & $\mathrm{y}$ & 192.7 & 0.0637 & $1.3:$ & $\mathrm{F} 6 \mathrm{~V}$ & 9.0 \\
\hline $16086-2540$ & TOK 726 & HD 144785 & 16.3876 & $\mathrm{I}$ & 63.7 & 1.8879 & $6.1 *$ & G8IV & \\
\hline $16315-3901$ & HDS $2335 \mathrm{Aa}, \mathrm{Ab}$ & HIP 80925 & 16.3907 & I & 68.6 & 0.0724 & 2.0 & $\mathrm{~K} 1 \mathrm{~V}$ & $44.5^{*}$ \\
\hline $16385+1240$ & TOK 727 & HIP 81476 & 17.4327 & I & 176.4 & 0.0876 & 2.1 & G0 & $10.2^{*}$ \\
\hline $17022-2820$ & TOK 728 & HD 153709 & 16.3876 & I & 164.4 & 0.6349 & 3.8 & $\mathrm{~A} 0 \mathrm{IV} / \mathrm{V}$ & $\ldots$ \\
\hline $17086-2650$ & SEE $319 \mathrm{Aa}, \mathrm{Ab}$ & HIP 83878 & 16.3876 & I & 40.2 & 0.0422 & 0.9 & B9IV & 3.5 \\
\hline $17095-2612$ & SKF $2521 \mathrm{Aa}, \mathrm{Ab}$ & CPD-26 5829 & 16.3876 & I & 161.0 & 0.1214 & $0.3:$ & $\mathrm{F} 8$ & $\ldots$ \\
\hline $17379-3752$ & I $247 \mathrm{AC}$ & HIP 86286 & 17.5345 & I & 350.5 & 0.1772 & 2.2 & G8IV & 21.0 \\
\hline $18086-2752$ & $\mathrm{BU} 244 \mathrm{Ba}, \mathrm{Bb}$ & HIP 88864 & 16.3877 & I & 146.1 & 0.1238 & 1.9 & G8III & 3.5 \\
\hline $19035-2645$ & $\mathrm{LDS} 5870 \mathrm{Aa}, \mathrm{Ab}$ & K 214324736 & 16.3882 & I & 66.8 & 0.5227 & $3.9:$ & G5V? & $9.4^{*}$ \\
\hline $19139-2548$ & B $2475 \mathrm{Aa}, \mathrm{Ab}$ & HD 179499 & 16.3880 & I & 118.3 & 0.3894 & $1.7 \mathrm{q}$ & F8 & $2.7^{*}$ \\
\hline $19164-2521$ & HJ $5101 \mathrm{Aa}, \mathrm{Ab}$ & HD 180132 & 16.3880 & I & 127.1 & 0.6919 & 4.4 & $\mathrm{~B} 9 / \mathrm{A} 0 \mathrm{~V}$ & $2.8^{*}$ \\
\hline $19197-2836$ & $\mathrm{~B} 433 \mathrm{AC}$ & HIP 94985 & 16.3880 & I & 51.7 & 2.4366 & 3.9 & G1V & 8.4 \\
\hline $19231-2833$ & RSS $520 \mathrm{Aa}, \mathrm{Ab}$ & HIP 95278 & 16.3880 & I & 156.6 & 0.3490 & $2.8:$ & F0 & 1.3 \\
\hline $19239-2939$ & HJ $5110 \mathrm{Aa}, \mathrm{Ab}$ & CD-29 16082 & 16.3880 & I & 215.8 & 0.1166 & $0.9:$ & $\mathrm{F} 4 \mathrm{~V}$ & $1.2^{*}$ \\
\hline $19391-2811$ & $\mathrm{~B} 444 \mathrm{Aa}, \mathrm{Ab}$ & HD 185233 & 16.3880 & I & 27.3 & 0.1002 & $1.5:$ & A5III & $\ldots$ \\
\hline $20100-1303$ & TOK 729 & HIP 99357 & 17.8246 & I & 277.2 & 1.2429 & $6.1 *$ & F5V & 4.4 \\
\hline $21012-3511$ & TOK $344 \mathrm{Aa}, \mathrm{Ab}$ & HIP 103735 & 17.6025 & I & 168.0 & 0.1747 & 1.7 & G3V & 21.5 \\
\hline $20212+0249$ & TOK 730 & HIP 103735 & 16.3900 & I & 143.85 & 0.2269 & 2.3 & G0 & 2.3 \\
\hline $21266-4604$ & HJ $5267 \mathrm{Aa}, \mathrm{Ab}$ & HIP 105879 & 17.6027 & I & 53.6 & 0.0874 & 1.9 & $\mathrm{~F} 7 \mathrm{~V}$ & 15.8 \\
\hline $21278-5922$ & TOK 731 & HIP 105976 & 16.3883 & I & 27.3 & 0.1002 & 1.5 : & F2IV & 8.0 \\
\hline $21357-5942$ & TOK 732 & HIP 106615 & 16.3901 & I & 185.3 & 0.7033 & $3.3 \mathrm{q}$ & G0V & 12.3 \\
\hline $23005-3345$ & TOK 733 & HIP 113598 & 17.8248 & I & 62.9 & 0.0634 & 1.4 & G4V & $12.3^{*}$ \\
\hline
\end{tabular}

and was unresolved in 2017.37; $P^{*}=13 \mathrm{yr}$.

HIP 70693 (HD 126620) is a new $0^{\prime \prime} .05$ pair with $P^{*}=$ $8 \mathrm{yr}$. Its re-observation shows decreasing separation at nearly constant PA.

HIP 72492 (HD 130264) is the Hipparcos pair HDS 2092 revealed here as a new triple system; we resolve the secondary into a $0 . \prime 057$ pair BC and observe its direct motion; $P_{\mathrm{RC}}^{*}=20 \mathrm{yr}$. Like some other triple dwarfs (Tokovinin 2018c), this is a "double twin": the estimated masses of $\mathrm{B}$ and $\mathrm{C}$ are about $0.8 \mathcal{M}_{\odot}$ each, and their sum is close to the mass of the main component $\mathrm{A}$, $1.6 \mathcal{M}_{\odot}$.

HIP 77843 (HD 142269) is a new 0 "' 13 pair with a fast motion, $P^{*}=21 \mathrm{yr}$.

HIP 78475 (HD 143235) is a 0.7 neglected binary SEE 254 observed as a "filler" to the main program. We discovered it to be a triple system where the primary component is a $0 . \prime 064$ pair. The separation implies $P^{*} \sim 12$ yr (we do see some motion in one month). The orbit of the subsystem could be computed now from the "wobble" in the trajectory of the outer pair if accurate measurements were available. Unfortunately, this is not the case.

$J 16086-2540$ (HD 144785) in the $\mathrm{K} 2$ field is resolved at 1 ". $89, \Delta I=6.1 \mathrm{mag}$; the $0 . .4$ Tycho pair TDS 1977 is spurious, see Section 3.2

HIP 80925 (HD 148704), a nearby K1V star with fast $\mathrm{PM}$, is a spectroscopic binary with $P=31.8$ days. The newly resolved pair $\mathrm{Aa}, \mathrm{Ab}$ has $P^{*}=1.5 \mathrm{yr}$; it should be detectable by $\mathrm{RV}$ variation. On the other hand, the $4^{\prime \prime}$ binary HDS 2335 is optical; the companion B is seen in 2MASS at 9 .' $^{\prime \prime}, 25^{\circ} .9$.

HIP 81476 (HD 150122) was known to be a binary from its variable RV and astrometric acceleration. It belongs to the 67-pc sample of nearby dwarfs, although the Gaia parallax of 10.2 mas puts it now outside $67 \mathrm{pc}$. D. Latham (2012, private communication) computed an unpublished 9 yr spectroscopic orbit. The star was observed at SOAR and unresolved in 2014.30, but now it is securely resolved at $0 . \prime 088$ separation. With $\Delta I=2.1$ mag, double lines should be detectable in the spectrum, opening the prospect of accurate mass measurement. The star is on the California program of planet search (Isaacson \& Fischer 2010). 
J17022-2820 (HD 153709) is a new pair with 0.63 separation and $\Delta I=3.8 \mathrm{mag}$ (below the estimated $5 \sigma$ detection limit of $3.4 \mathrm{mag}$ ). It was observed as the Tycho binary TDT 186, which is revealed to be spurious, see Section 3.2

HIP 83878 (SEE 319) is a B9IV star with a slow PM. The status of the known companion at $7^{\prime \prime} .7$ is uncertain (it can be optical). The newly detected subsystem Aa, Ab at $0 . \prime 042$, confirmed a year later, has an estimated period of $\sim 20 \mathrm{yr}$.

$C P D-265829$ (SKF 2521) is another wide 7.4 binary from the K2 program where we found a subsystem $\mathrm{Aa}, \mathrm{Ab}$. With the photometric parallax of 3.2 mas, the estimated period of $\mathrm{Aa}, \mathrm{Ab}$ is over $100 \mathrm{yr}$; no motion is detected in one year.

HIP 86286 is a known visual binary I 247 . Unexpectedly, we found two companions $\mathrm{B}$ and $\mathrm{C}$ of similar brightness in a nearly equilateral configuration, both at $0^{\prime \prime} 2$ separation from the primary. In 1897 the separation was $1^{\prime \prime}$. It steadily decreased during the 20th century, in agreement with the long estimated period $P^{*}=200 \mathrm{yr}$. Presumably, the secondary component is a close binary $\mathrm{BC}$, not recognized until now. This object resembles the similar equilateral triple I 213 discovered at SOAR in 2015 (Tokovinin et al. 2016a).

HIP 88864 (HD 165732) is a G8III giant. In addition to the known $2 . .2$ pair BU 244, we measured the subsystem $\mathrm{Ba}, \mathrm{Bb}$ at 0 .'12. Re-observation after one year revealed its retrograde motion, although the parallax of 3.5 mas implies $P_{\mathrm{Ba}, \mathrm{Bb}}^{*}=120 \mathrm{yr}$.

EPIC 214324736 (TYC 6881-1560-1) is the $17^{\prime \prime} 4$ CPM pair LDS 5870 with the Gaia parallax of 9.4 mas. The components' PMs and photometric distances match. WDS lists a discordant first measure in 1960 which, if true, would mean that AB is optical; we believe the first measure to be in error because the configuration of $\mathrm{AB}$ remained fixed for 15 years between its measurements by 2MASS and Gaia. The newly discovered 0.52 subsystem $\mathrm{Aa}, \mathrm{Ab}$ is also fixed, proving its physical nature; $P_{\mathrm{Aa}, \mathrm{Ab}}^{*}=330 \mathrm{yr}$.

$H D 179499$ is the known visual pair B 2475 at $8^{\prime \prime} .2$ separation. The Gaia parallax of the secondary component is 2.7 mas, while it gives no parallax for the primary, possibly because it was resolved. Indeed, we discovered $\mathrm{Aa}, \mathrm{Ab}$ at $0 . .39$; its position did not change in a year. Its estimated orbital period is $\sim 1$ kyr. However, the F8 spectral type of $\mathrm{A}$ and the colors of both $\mathrm{A}$ and $\mathrm{B}$ indicate a photometric parallax of 5 mas, inconsistent with the Gaia parallax.

HD 180132 is a $21^{\prime \prime} 4$ pair HJ 5101 AB. Matching Gaia parallaxes and PMs of both components leave no doubt that this pair is physical, despite its first discordant measure in WDS. The companion to A discovered here at 0.69 is probably too faint to be detected by Gaia ( $\Delta I=4.4 \mathrm{mag}$ ). The period of $\mathrm{Aa}, \mathrm{Ab}$ is long, $\sim 2 \mathrm{kyr}$; its position is fixed during one year.

HIP 94985 is a tight 0.2 binary B 433 to which we discovered a faint $(\Delta I=3.9 \mathrm{mag})$ companion at $2^{\prime \prime} .44$. If the companion were a distant unrelated star with a zero PM, the separation of AC would have increased by 38 mas in one year. Instead, we found it to be constant to within 3 mas. The 0.4 change in the PA during this period is likely related to the angle calibration problems in run 3.

HIP 95278 is a $9 . ! 7$ pair RSS 520 located in a relatively crowded region of the sky; its faint secondary component is likely optical. The small PM and parallax do not help in defining its status. The newly discovered subsystem $\mathrm{Aa}, \mathrm{Ab}$ at 0.39 has a better chance to be physical, but its long period of $\sim 2.5$ kyr does not inspire any interest in this discovery.

CD-29 16082 (HJ 5110) is a 5".9 near-equal pair where we found a 0 "! 12 subsystem Aa,Ab. This triple system could be physical. However, the Gaia parallax of 1.2 mas implies very long periods; if the parallax is correct, both components $\mathrm{A}$ and $\mathrm{B}$ are located above the main sequence.

HD 185233 is a $1^{\prime \prime}$ binary B 444 without trigonometric parallax or orbit. We discovered the $0^{\prime \prime} .10$ subsystem Aa,Ab. This resolution, although not checked in 2017, is secure. The expected period of $\mathrm{Aa}, \mathrm{Ab}$ is a couple of centuries, given the photometric parallax of 1.9 mas.

HIP 99357 (HD 191365) is an F5V spectroscopic binary according to the GCS, based on two mutually discordant measures of the RV. The new faint companion at $1^{\prime \prime} .34$ with $\Delta I=6.1$ mag may be optical.

HIP 100355 was pointed in 2015 by mistake instead of HLD 158 (WDS J20213+0250) and resolved at 0 '!2, in marked disagreement with the orbit of HLD 158 (Tokovinin et al. 2016a). Now the confusion is clarified, as both HIP 100355 and HLD 158 were measured. Our published 2015 measure should be attributed to HIP 100355.

HIP 103735 (HD 199918) is a nearby G3V star with a wide $\left(186^{\prime \prime}\right)$ CPM companion, which possibly is a white dwarf. The main star is a spectroscopic and astrometric binary, first resolved here at 0.17 . The separation implies an orbital period of the order of $20 \mathrm{yr}$ that might explain the non-resolution of this binary at Gemini in 2011 (Tokovinin et al. 2012).

HIP 105879 (HD 203934) has astrometric acceleration and variable RV (CGS). Its spectrum has double lines, according to the ongoing monitoring at the CTIO $1.5 \mathrm{~m}$ telescope. The first attempts to resolve the pair at Gemini (Tokovinin et al. 2013) and at SOAR in 2015.74 were unsuccessful, but in 2017.6 it was securely resolved at 0 !'087. The separation and magnitude difference match the preliminary spectroscopic orbit with $P=5 \mathrm{yr}$ and the mass ratio $q=0.78$. According to this orbit, the separation in 2015.74 was 0 .'025. Observations for several more years will lead to accurate measurements of the masses of components $\mathrm{Aa}$ and $\mathrm{Ab}$; the next periastron is predicted in 2020.6. The CPM companion D at $44^{\prime \prime}$ makes this system triple. The star D has a constant $\mathrm{RV}$ and it has not been resolved at SOAR in 2014.

HIP 105976 (HD 203970) is resolved at 0".09 in 2016.4; it moved by $8^{\circ}$ in one year, matching the estimated pe$\operatorname{riod} P^{*}=30 \mathrm{yr}$.

HIP 106615 (HD 205158) has a new faint companion at 0.70 with $P^{*}=300 \mathrm{yr}$. The star is on the Magellan planet search program.

HIP 113598 (HD 217344) is a $3 .^{\prime \prime} 9$ physical pair B 582 containing a 1.6 day SB1 TZ PsA. We tentatively resolved an intermediate subsystem Aa, Ab at 0".063. However, this result needs confirmation because telescope vibrations could mimic the resolution. If this pair is real, its period is short, $P^{*}=8 \mathrm{yr}$. 


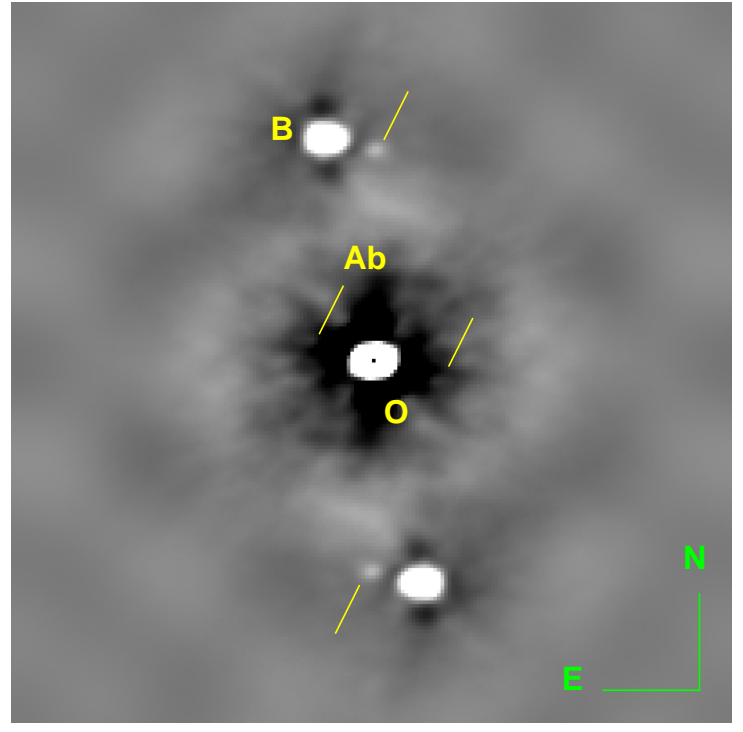

Figure 4. ACF of BU 83 recorded on 2017.83 in the $I$ band. The coordinate center is at $\mathrm{O}$, the letters indicate the two peaks corresponding to $\mathrm{B}$ and $\mathrm{Ab}$, while lines mark all four peaks caused by the faint component $\mathrm{Ab}$ (the two central peaks are lost in the noise).

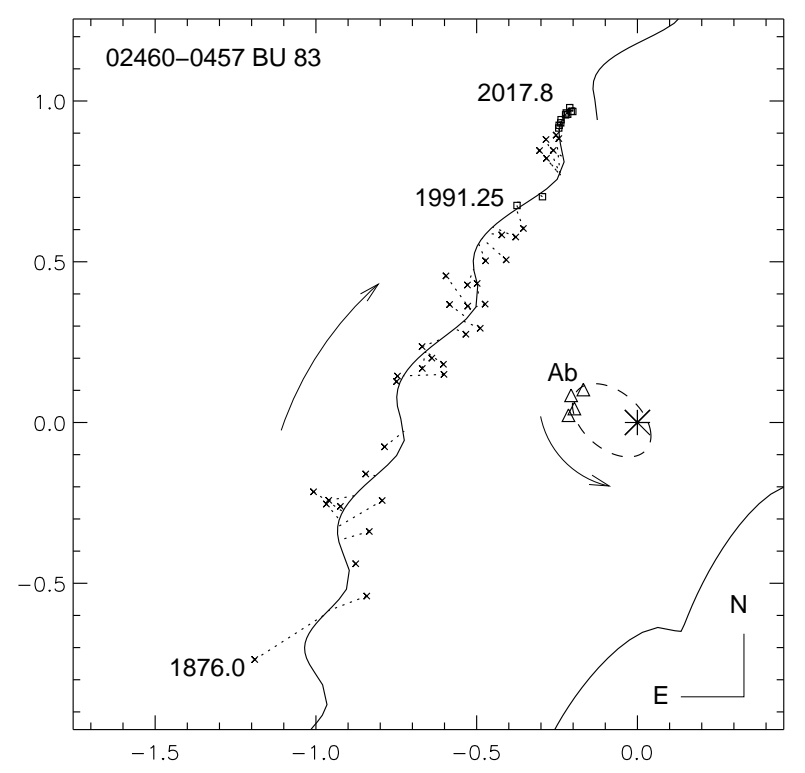

Figure 5. Trajectory of the outer subsystem in BU 83 accounting for the wobble caused by the inner subsystem. Crosses - micrometer measures and speckle interferometry at small apertures, squares - accurate recent measures at SOAR and Hipparcos, line - the orbit. The dashed ellipse and triangles denote the orbit and resolved measures of the subsystem $\mathrm{Aa}, \mathrm{Ab}$. The axis scale is in arcseconds.

\subsection{The triple system $B U 83$}

One of the calibrator binaries, WDS J02460-0457 (BU 83, HIP 12912, ADS 2111, F3V), is found to be triple. Looking back at the archival SOAR data, the tertiary component can be noted in several $I$-band ACFs. The clearest previous detection was in 2012.92, overlooked at the time. The inner pair $\mathrm{Aa}, \mathrm{Ab}$ was then at $58^{\circ} .7,0^{\prime \prime} \cdot 20, \Delta I=4.5 \mathrm{mag}$; it turned by $23^{\circ}$ in 5 years. A very tentative measure of $\mathrm{Aa}, \mathrm{Ab}$ in 2009.671 is $58^{\circ} .9$, 0 "'19. In 2015, the companion's detection was marginal, but it was resolved securely in 2016 and 2017 (Figure 4). The companion is not seen in the $y$ band. Owing to the faintness of the companion Ab, the fitted triple-star models do not converge well, resulting in the low accuracy of the measures of $\mathrm{Aa}, \mathrm{Ab}$.

Interestingly, the existence of the subsystem was suspected by Dommanget (1972) from the run of the residuals of the outer pair. He proposed a $36 \mathrm{yr}$ astrometric orbit with an amplitude of 0. . 08 .

Figure 5 shows that the accurate SOAR measures indeed deviate from the long-period orbit (which itself is poorly defined by the short observed arc). Using the orbit3.pro code $^{4}$ (Tokovinin \& Latham 2017), we can fit the wobble and the resolved measures of $\mathrm{Aa}, \mathrm{Ab}$ by two sets of orbital elements. However, the low accuracy of historic measures and the partial coverage of the inner resolved orbit make such fits rather uncertain. The plots in the Figure correspond to the inner period of 38 yr and the outer period of $915 \mathrm{yr}$. The inner subsystem has direct motion, while the outer orbit is retrograde, so the angle between the inner and outer angular momentum vectors is large. The sign of the wobble confirms that the subsystem belongs to the primary component. Patient accumulation of measures is needed before attempting a meaningful analysis of this interesting triple system. The intuition of J. Dommanget who discovered the wobble in the noisy measures available prior to 1972 is truly amazing, but the astrometric orbit derived from the old data alone cannot be trusted.

We thank the operators of SOAR D. Maturana (he passed away in 2017), P. Ugarte, S. Pizarro, J. Espinoza, C. Corco, R. Hernandez for efficient support of our program, R. Cantarutti for adapting the HRCam software to the new detector, and N. Law for offering us his iXon- 888 camera. Some observations were made using the Luca-R cameras borrowed from G. Cecil and from the STELES team, and we are thankful for this. Detailed comments by the Referee helped us to improve the presentation and the tables.

R.A.M. acknowledges support from the Chilean Centro de Excelencia en Astrofísica y Tecnologías Afines (CATA) BASAL PFB/06, the Project IC120009 Millennium Institute of Astrophysics (MAS) of the Iniciativa Científica Milenio del Ministerio de Economía, Fomento y Turismo de Chile, and CONICYT/FONDECYT Grant Nr. 1170854.

This work used the SIMBAD service operated by Centre des Données Stellaires (Strasbourg, France), bibliographic references from the Astrophysics Data System maintained by SAO/NASA, and the Washington Double Star Catalog maintained at USNO.

Facilities: SOAR.

\section{REFERENCES}

Briceño, C. \& Tokovinin, A. 2017, AJ, 154, 195

Dommanget, J. 1972, Bull. RAS Belg., 8, 63

Gaia Collaboration, Brown, A. G. A., Vallenari, A., Prusti, T. et al. 2016, A\&A, 595, 2

Gomez, J., Docobo, J. A., Campo, P. P., \& Mendez, R. 2016, AJ, 152,216

\footnotetext{
4 http://dx.doi.org/10.5281/zenodo.321854
} 
Hartkopf, W. I., Mason, B. D. \& Worley, C. E. 2001, AJ, 122, 3472

Hartkopf, W. I., Tokovinin, A. \& Mason, B. D. 2012, AJ, 143, 42 Horch, E. P., van Belle, G. T., Davidson, J. W., Jr. et al. 2015, AJ, 150, 151

Horch, E. P., Casetti-Dinescu, D. I., Camarata, M. A. et al. 2017, AJ, 153, 212

Isaacson, H. \& Fischer, D. 2010, ApJ, 725, 875

Makarov, V. V. \& Kaplan, G. H., 2005, AJ, 129, 2420

Mason, B. D., Wycoff, G. L., Hartkopf, W. I. et al. 2001, AJ, 122, 3466 (WDS)

Mason, B. D., Hartkopf, W. I., Miles, K. N., Subasavage, J. P., Raghavan, D. \& Henry, T. J. 2018, AJ (in press)

Mendez, R. A., Claveria, R. M., Orchard, M. E., \& Silva, J. F. 2017, AJ, 154, 187

Muterspaugh, M. W., Hartkopf, W. I., Lane. B. F. et al. 2010a, AJ, 140, 1623

Muterspaugh, M. W., Fekel, F. C., Lane, B. F. et al. 2010b, AJ, 140,1646

Nidever, D.L., Marcy, G.W., Butler, R.P. et al. 2002 ApJS, 141, 503

Nordström, B., Mayor, M., Andersen, J. et al. 2004, A\&A, 418, 989 (GCS)

Sauvage, J.-F., Fusco, T., Guesalaga, A. et al. 2015, in Proceedings of the Conference "Adaptive Optics for Extremely Large Telescopes 4."

http://www.escholarship.org/uc/item/910646qf

Tokovinin, A. \& Cantarutti, R. 2008, PASP, 120, 170
Tokovinin, A., Mason, B. D., \& Hartkopf, W. I. 2010a, AJ, 139, 743 (TMH10)

Tokovinin, A. Cantarutti, R., Tighe, R. et al. 2010b, PASP, 122 , 1483

Tokovinin, A. 2012, AJ, 144, 56

Tokovinin, A. Hartung, M., Hayward, Th. L., \& Makarov, V. V. 2012, AJ, 144, 7

Tokovinin, A. Hartung, M., Hayward, Th. L. 2013, AJ, 146, 8

Tokovinin, A. 2014, AJ, 147, 86

Tokovinin, A., Mason, B. D., \& Hartkopf, W. I. 2014, AJ, 147, 123

Tokovinin, A., Mason, B. D., Hartkopf, W. I. et al. 2015, AJ, 150, 50 (SOAR14)

Tokovinin, A., Mason, B. D., Hartkopf, W. I. et al. 2016a, AJ, 152,116

Tokovinin A., Cantarutti, R., Tighe R., et al. 2016b, PASP, 128, 125003

Tokovinin, A. 2016, AJ, 152, 138

Tokovinin, A. \& Latham, D. W. 2017, ApJ, 838, 54

Tokovinin, A. 2017, AJ, 154, 110

Tokovinin, A. 2018, PASP, 130, 5002

Tokovinin, A. 2018a, Inf. Circ. 194, 1

Tokovinin, A. 2018c, AJ, in press (DOI

https://doi.org/10.3847/1538-3881/aab102)

van Leeuwen, F. 2007, A\&A, 474, 653 